\title{
Texture and Strain Measurements from Bending of NiTi Shape Memory Alloy Wires
}

\author{
Matthew Carl ${ }^{1} \cdot$ Baozhuo Zhang ${ }^{1} \cdot$ Marcus L. Young ${ }^{1}$
}

Published online: 19 July 2016

(C) ASM International 2016

\begin{abstract}
Shape memory alloys (SMAs) are a new generation of materials that exhibit unique nonlinear deformations due to a phase transformation which allows the material to return to its original shape after removal of stress or a change in temperature. These unique properties are the result of a martensitic/austenitic phase transformation through the application of temperature changes or applied stress. Many technological applications of austenitic SMAs involve cyclical mechanical loading and unloading in order to take advantage of pseudoelasticity, but are limited due to poor fatigue life. In this paper, commercial pseudoelastic NiTi SMA wires (50.7 at.\% Ni) were placed under different bending strains and examined using scanning electron microscopy and high-energy synchrotron radiation X-ray diffraction (SR-XRD). By observing the microstructure, phase transformation temperatures, surface texture and diffraction patterns along the wire, it is shown that the wire exhibits a strong anisotropic behavior whether on the tensile or compressive side of the bending axis and that the initiation of micro-cracks in the wires is localized on the compression side, but that crack propagation will still happen if the wire is reloaded in the opposite direction. In addition, lattice strains are examined for both the austenite and martensite phases.
\end{abstract}

This article is an invited paper selected from presentations at the International Conference on Shape Memory and Superelastic Technologies 2015, held May 18-22, 2015, in Chipping Norton, Oxfordshire, United Kingdom.

Matthew Carl

matthewcarl@my.unt.edu

1 Department of Materials Science and Engineering, University of North Texas, Denton, TX 76207, USA
Keywords Bending · Pseudoelastic - Synchrotron X-ray diffraction $\cdot$ Shape memory alloys

\section{Introduction}

The shape memory effect (SME) was first observed in materials (Au-Cd and In-Tl [1, 2]) in the early 1930s. In 1963, a metallurgist at the Naval Ordnance Laboratory discovered that an alloy of nickel and titanium, hence the name Nitinol, exhibited excellent fatigue resistance and unique mechanical properties such as the SME and pseudoelasticity (PE) $[3,4]$. The specific property is dependent upon the initial condition (martensite or austenite phase) and the thermomechanical mechanism for the phase transformation. In general, stress-induction and/or thermal changes are the main methods used to induce the phase transformation in order to take advantage of SMAs' unique mechanical properties. This phase transformation gives NiTi SMAs the ability to recover strains on the order of 5-10\%, an order of magnitude higher than the elastic strain recovery of typical metals. Besides their good mechanical properties, NiTi SMAs also have good chemical properties, abrasion resistance, and corrosion resistance [5-9].

Many experiments involving the study of these unique properties of NiTi shape memory alloys (SMAs) have taken place since 1963 and as a result of these studies, NiTi SMAs have been used for many applications, but one major concern still is their fatigue life $[10,11]$. The fatigue life of pseudoelastic NiTi SMAs specifically has been studies by a number of research groups. Eggeler et al. [12] examined pseudoelastic NiTi SMA wires through low cycle pull-pull and bending-rotation fatigue (BRF) of wires and observed strain localization during the phase transformation. Their 
results show that irreversible strain accumulates during testing in BRF experiments and that the surface cracks after increasing the cycling load stress which governs the fatigue life. Surface cracking was also observed by James et al. [13] when pseudoelastic NiTi SMA wires were subjected to high strain levels on the compression side in bending and showed compressive damage failure mode occurs in these wires when the bending strain is higher than $20 \%$. Synchrotron radiation X-ray diffraction (SR-XRD) during in situ mechanical loading is a useful tool in understanding the phase behavior and therefore the mechanical response of pseudoelastic NiTi wires. Schmahl et al. [14] studied the phase evolution of a pseudoelastic NiTi SMA during tensile loading using SR-XRD and showed that a "highstress" austenite region is formed in a shear-band at the transformation interface. Hasan et al. [15] further examined NiTi in uniaxial mechanical loading by showing the texture evolution of the martensite variants using 2D diffraction images obtained with transmission high-energy SR-XRD. Young et al. [16] performed another detailed study using high-energy SR-XRD by examining the phase transformation behavior of a fine-grained pseudoelastic NiTi SMA wire in uniaxial loading and unloading. Their results further proved by examination of zone width and phase volume fraction that the localization of the phase transformation occurs while also suggesting reasons for the nonlinear elasto-plastic deformation behavior observed in pseudoelastic NiTi SMA wires through macro- and microstrain measurements. Recently Cai et al. [17] used SR-XRD to study the effects of heat-treating temperature of NiTi SMA wires on the mechanical properties and concluded that fatigue life could be improved with low temperature heat treatments by lowering internal strain in the austenite phase. Considering these studies and the industrial need to improve the fatigue life in pseudoelastic NiTi SMAs under bending, we use high-energy SR-XRD during in situ simple bending of an austenitic NiTi (50.7 at.\% Ni) SMA wire to examine phases, texture, and local residual microstrains in the wire. In addition, the evolution of micro-cracks along the surface are examined using scanning electron microscopy (SEM).

\section{Experimental Methods}

Austenitic pseudoelastic NiTi (50.7 at.\% Ni) SMA wires (Nitinol Devices and Components) with a diameter of $1.26 \mathrm{~mm}$ were examined in simple bending using SEM and high-energy SR-XRD. Some of the NiTi SMA wires were heat-treated at $500{ }^{\circ} \mathrm{C}$ for $30 \mathrm{~min}$ to form $\mathrm{Ni}_{4} \mathrm{Ti}_{3}$ precipitates, which contribute to inducing the complete phase transformation and improve the fatigue life. A Netzsch 204F1 Phoenix differential scanning calorimeter (DSC) was used to measure transformation temperatures. The temperature range was cycled between $-25^{\circ} \mathrm{C}$ to $65^{\circ} \mathrm{C}$ with a heating/cooling rate of $10^{\circ} \mathrm{C} / \mathrm{min}$ and held for $5 \mathrm{~min}$ at the maximum and minimum temperature to allow the machine to stabilize before heating or cooling. $40 \mathrm{~mm}$ gage lengths of NiTi SMA wires were prepared for the bending experiments. The wires were progressively bent with different radii of curvature, $0,2.1$ and $5.25 \mathrm{~mm}$, which correspond to $0 \%$ surface strain, $12 \%$ surface strain, and $30 \%$ surface strain, respectively. These surface strains were calculated according to Wagner et al. [18] using the following equation: $\varepsilon_{\mathrm{a}}=d / 2 R$, " $\varepsilon_{\mathrm{a}}$ " for the strain amplitude where " $R$ " represents the radius of the semicircle, and " $d$ " is the diameter of the wire. Samples were electrochemically polished at $15 \mathrm{~V}$ for $60 \mathrm{~s}$ at room temperature using a Struers LectroPol-5 electro-polisher in a solution of $20 \% \mathrm{H}_{2} \mathrm{SO}_{4}$ and $80 \%$ methanol. SEM images were collected before and after bending using a FEI Quanta 200 ESEM equipped with a scanning electron microscopy (EDS) system. Further examination of the $12 \%$ surfacestrained wire was performed in beam line 1-ID-C at the advanced photon source (APS) in Argonne National Laboratories (ANL); experimental setup presented in Fig. 1. SR-XRD patterns were collected during in situ bending of the $12 \%$ surface-strained wire at a beam energy of $70 \mathrm{keV}$, an exposure time of $1 \mathrm{~s}$, and a beam size of $200 \times$ $200 \mu^{2}$. The subsequent Debye-Scherrer diffraction cones were then collected on a G.E. 2D detector plate set at a distance of approximately $1.377 \mathrm{~m}$ from the wire sample. Customized MATLAB code was used to analyze the SRXRD patterns [16, 19], Fit2D software was used to convert diffraction patterns from polar to Cartesian coordinates [20], and diffraction peaks were identified using the crystallographic parameters from power diffraction files for austenite (PDF \# 03-065-5537), martensite (PDF \# 03-0650145), and R-phase [21]. These values were further refined by generating lattice spacing versus $\sin ^{2} \psi$ plots when possible. Methods as those previously mentioned are fully described in Ref. [16, 19, 21, 22] and have been used to determine lattice strains of the phases present. Throughout the text and figures the lattice strains are presented in microstrain $(\mu \varepsilon)$, which is merely the absolute strain value multiplied by a factor of $10^{6}$. This allows for easy comparison of the magnitude across the sample at different positions.

\section{Results and Discussion}

\section{Microstructure and DSC Measurements}

Figure 2a, b shows SEM images from the as-received and heat-treated NiTi SMA wire, respectively. The 
Fig. 1 Schematic of experimental setup and parameters used during synchrotron radiation X-ray diffraction measurements taken at beamline $1-\mathrm{ID}-\mathrm{C}$ at the Advanced Photon Source (APS) in Argonne National Laboratories (ANL)

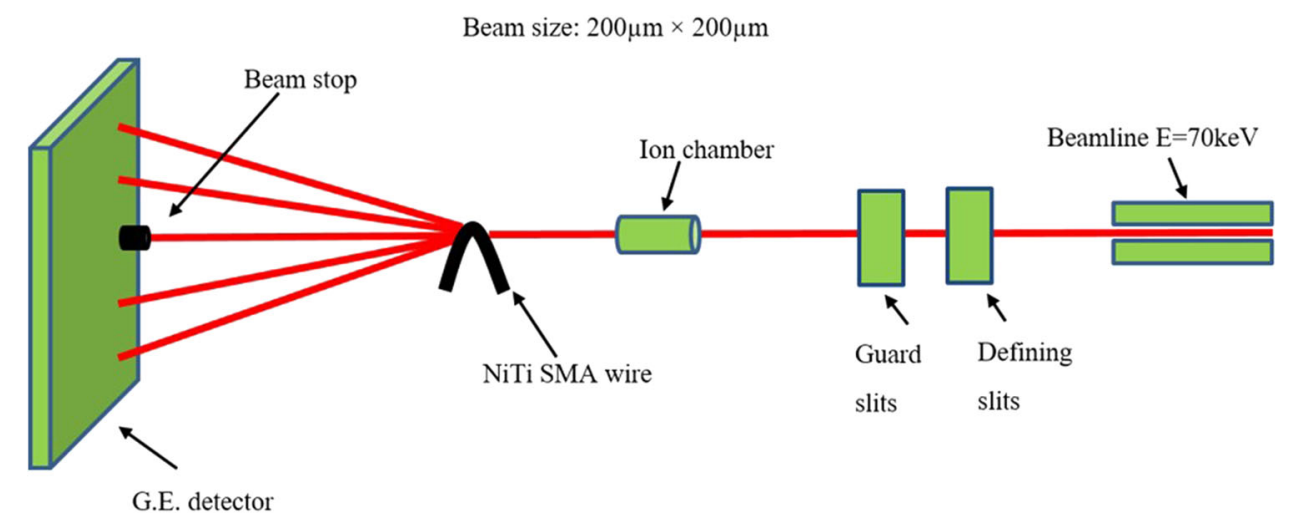

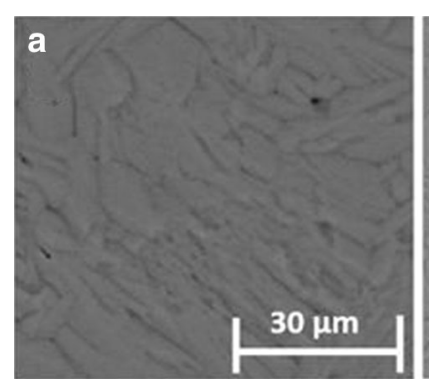

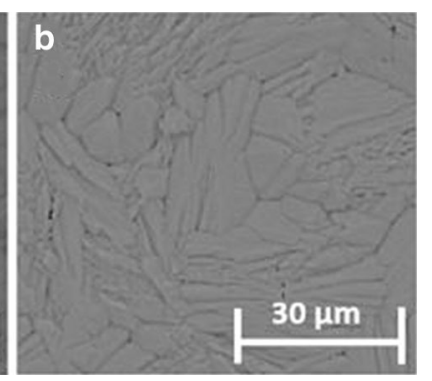

Fig. 2 Secondary electron microscopy images of the a as-received and $\mathbf{b}$ heat-treated NiTi SMA wire

microstructure for both conditions looks very similar with some large equi-axed grains with an average grain size of about $10-15 \mu \mathrm{m}$ and some smaller elongated lathe-like grains. Nanometer-sized $\mathrm{Ni}_{4} \mathrm{Ti}_{3}$ precipitates are expected to form during the heat treatment of the Ni-rich NiTi SMA wire, which induce a localized residual strain within grains that help promote the phase transformation. While these small precipitates are not observable here due to the low magnification of the SEM images, DSC curves (Fig. 3) show that a change has occurred between the as-received and heat-treated NiTi SMA wire, presumably due to the recovery of plastic deformation due to processing and the formation of these precipitates. The as-received NiTi SMA wire exhibits no transformation peaks in the range tested, as the phase transformation from austenite-to-martensite is likely suppressed due to large plastic deformation during the drawing process, i.e., cold working, while the heattreated NiTi SMA wire exhibits a reversible one-step transformation in the test range. However, due to the narrow hysteresis width associated with the two transformation peaks, approximately $20.2{ }^{\circ} \mathrm{C}$, it is unlikely that the transformation during cooling is of the normal B2-B19' phase transformation associated with a single-step phase transformation but rather is fully consistent with the intermediate B2-R transformation [23]. This type of transformation behavior is induced due to local

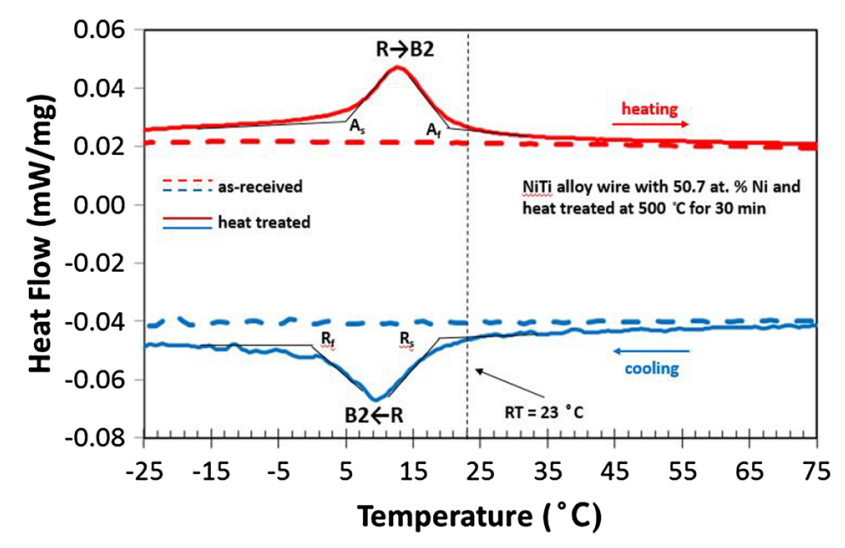

Fig. 3 DSC curves for the as-received (dotted lines) and heat-treated (solid lines) NiTi SMA wires

compositional changes and lattice strains caused by the formation of $\mathrm{Ni}_{4} \mathrm{Ti}_{3}$ precipitates in the material. In addition, Xue et al. [23] show that the B2-R transformation is a function of annealing temperature, e.g., lower annealing temperatures result in higher R-phase transformation start temperatures $\left(R_{\mathrm{s}}\right)$, while the R-B19' ${ }^{\prime}$ phase transformation is a function of annealing time, e.g., longer times increase the martensitic start temperature $\left(M_{\mathrm{s}}\right)$; both of which are consistent with the results presented in Fig. 3, where the R-B19' transformation peak is most likely below the $-30{ }^{\circ} \mathrm{C}$ test range. For a further more rigorous discussion of this phase stability, the reader is referred to Ref. [23]. Applying this logic, the transformation peaks shown in Fig. 3 are labeled $\mathrm{R} \rightarrow \mathrm{B} 2$ and $\mathrm{B} 2 \rightarrow \mathrm{R}$, during heating and cooling, respectively. It is possible that during heating the material would display a single-step transformation, i.e., B19'-B2 due to the high annealing temperature, but since the temperature cycle never reached a low enough temperature to induce the formation of the monoclinic B19' phase it has been labeled as an R-B2 transformation peak. Regardless, the transformation temperatures for the austenite transformation start temperature $\left(A_{\mathrm{s}}\right)$, austenite transformation finish temperature $\left(A_{\mathrm{f}}\right), \quad$ R-phase 
transformation start $\left(R_{\mathrm{s}}\right)$, and R-phase transformation finish temperature $\left(R_{\mathrm{f}}\right)$ were determined to be $4.9,20.1,-0.1$, and $18.0^{\circ} \mathrm{C}$, respectively, for the heat-treated $\mathrm{NiTi}$ wire indicating that the wire is fully austenitic at room temperature.

\section{SEM Observation of Pure Bending}

The bending method and SR-XRD measurement locations are illustrated in Fig. 4, while Fig. 5 shows SEM images collected at both the outer region (hereafter referred to as the tensile side) and inner region (hereafter referred to as the compressive side) of the bending axis the heat-treated NiTi SMA wires at 0,12 , and $30 \%$ surface strain, respectively. As expected, the wire at $0 \%$ surface strain looks very smooth with no surface cracking apparent on either the tensile or compressive side of the wire. However, as the surface strain is increased to $12 \%$, the wire must accommodate both the tensile and the compressive strains imposed. Along the tensile side, the surface texture is rather smooth with a few streaks parallel to the drawing direction, which is also associated with the tensile forces due to bending. The distance between these streaks is approximately 30-60 $\mu \mathrm{m}$ decreasing in distance as the top of the wire is approached or as the distance from the center (neutral axis) is increased, where the tensile force would be the greatest. This puts the streaks on the order of the austenite grain size. Within these bands another substructure is apparent upon closer examination that can be described as slashes between the main streaks. These slashes correspond to the martensitic structure associated with the now stress-induced phase transformation in the wire. No micro-cracks are observed and it seems that the martensite can easily accommodate the deformed wire in tension through the multiple martensitic variants and twinning methods characteristic of NiTi SMAs. Looking at the compressive side, the behavior is slightly different.
Although there are still horizontal streaks, they are shorter in the drawing direction and appear more frequently. In addition, the texture appears to be of a diamond pattern or "scale" on the surface. Surface cracking is not apparent in the image, but this does not rule out the possibility of extremely fine micro-cracks starting at the surface. It is obvious that accommodation of strain associated with bending by the martensitic transformation is more difficult in the wire. This is evident by the complex diamond pattern rather than the streaking pattern in the tensile side. This diamond pattern is directly related to the twinning and variant structure in the martensitic matrix which is much more complex in the compressive side than in the tensile side, indicating that stress-induced martensitic transformation needs to form more complex geometries to accommodate for the compressive forces and corresponding macroscopic shortening in overall length. As the surface strain is increased to $30 \%$, the surface textures increase. On the tensile side, the streaking is more frequent and smaller in separation distance as the max radial distance from the center is approached and eventually almost resembling the diamond pattern in the compressive side of the $12 \%$ surface-strained wire. Interestingly no cracking is observed visually, but again this does not rule out the possibility of fine micro-cracks at the near-surface region. The compressive side at $30 \%$ surface strain reveals the root of the short cyclical fatigue life and eventual failing of NiTi SMA wires. Large micro-cracks (one over $200 \mu \mathrm{m}$ in length as seen in Fig. 5) are visible all along the bending axis running perpendicular to the drawing direction or the bending radius. The majority of the cracking are contained within the first $200 \mu \mathrm{m}$ of the surface and are approximately $20-50 \mu \mathrm{m}$ in length. However, the three large cracks have started to grow and will propagate through the wire until its eventual and inevitable failure which has already begun. The surface has delineated from itself and is hanging free in two locations from which the two largest

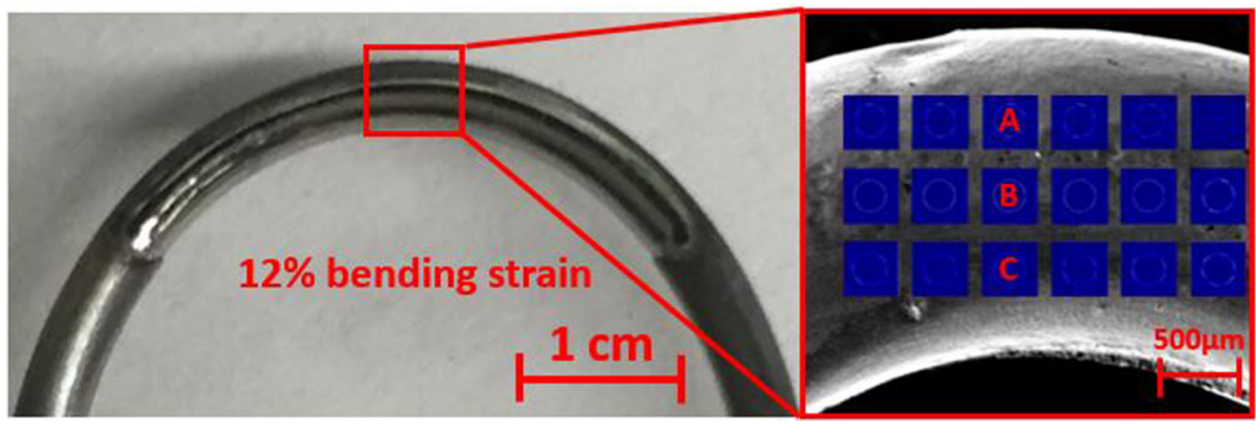

Fig. 4 Macroscopic image of an NiTi SMA wire at $12 \%$ bending strain and a zoomed in SEM image showing the approximate locations of the SR-XRD measurements. Points $A, B$, and $C$ refer to points in the mapping pattern where $\varepsilon_{11}$ and $\varepsilon_{22}$ are closest to $90^{\circ}$ and $0^{\circ}$, respectively. The highly reflective surface region in the macroscopic image is due to electro-polishing of the surface after SR-XRD measurements were taken to examine the surface texture in SEM 


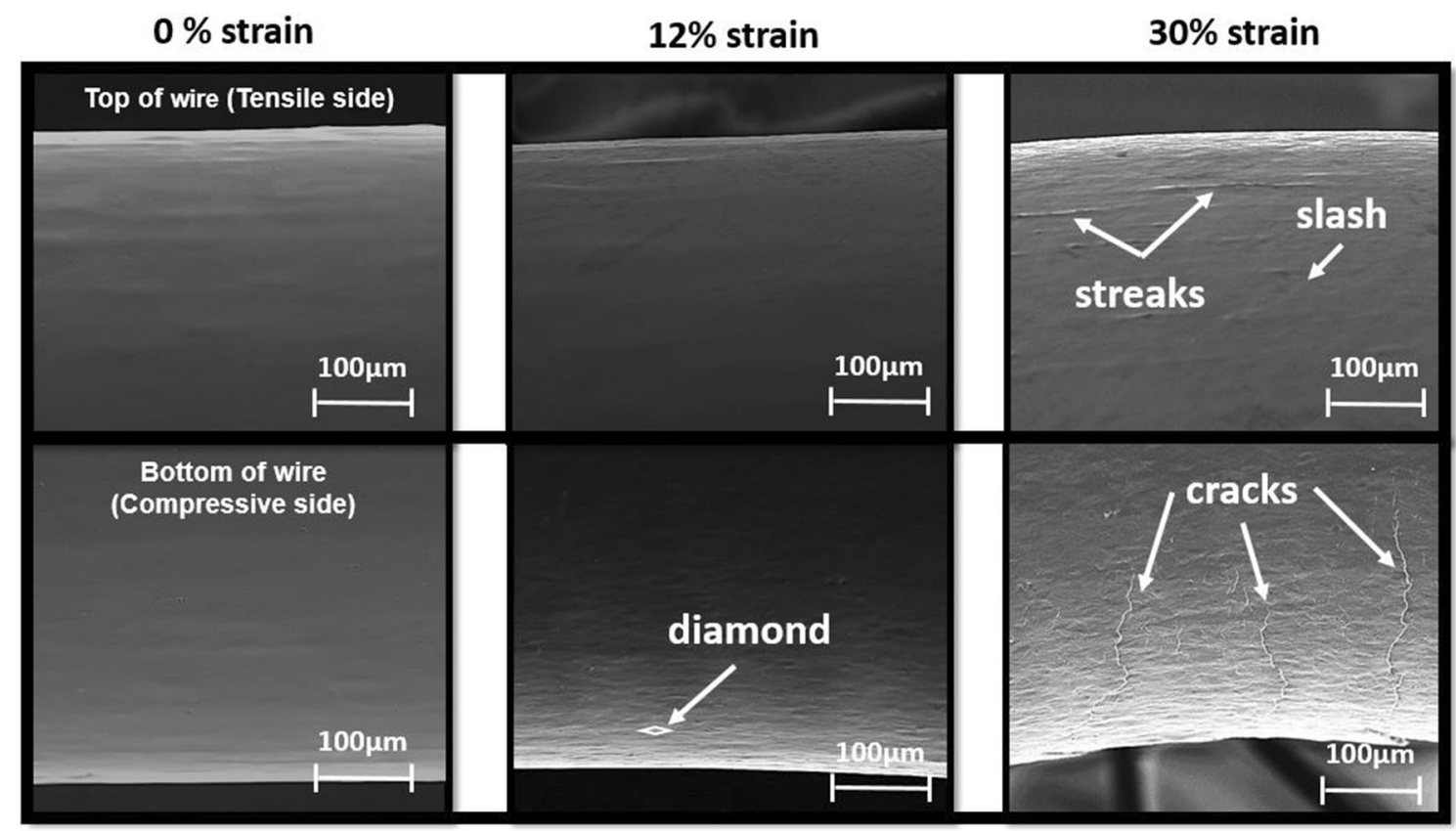

Fig. 5 Secondary electron SEM images of the surface texture along the tensile side, top images, and compressive side, bottom images, at bending strain values of 0,12 , and $30 \%$. Surface cracking is not apparent until $30 \%$ strain is reached and localized to the compressive side of the wire

remainder of this article $\varepsilon_{11}$ will refer to the strain in the direction perpendicular to the bending radius and $\varepsilon_{22}$ will refer to the strain that is tangential to the bending radius. These values also correspond to minima and maxima in the sinusoidal-like curves of the calculated strains for each $(\mathrm{hkl})$ plane as a function of azimuthal angle in the DebyeScherrer rings, example shown in Fig. 7 for the austenite (100) peaks on the center row of patterns in Fig. 6.

Integrated lineouts over the entire Debye-Scherrer rings for points $\mathrm{A}, \mathrm{B}$, and $\mathrm{C}$ are presented in Fig. 8. Indexing of the diffraction patterns show that both points $\mathrm{A}$ and $\mathrm{C}$ appear to be fully martensitic due to the stress associated with $12 \%$ bending strain. Point $\mathrm{B}$, the neutral axis, however is more complex. Although the wire is mainly austenite, small amounts of martensite and R-phase are measureable in the diffraction pattern. This becomes more evident when plotting the Cartesian transform of the full Debye-Scherrer rings with respect to azimuthal angle versus d-spacing, Fig. 9. While R-phase does not appear in the DSC curves at room temperature (Fig. 3), stress-induced R-phase peaks are clearly seen here rather than being washed out by the much more intense austenite (110) peak. The presence of the R-phase indicates that a certain level of stress has been reached which is below the martensitic phase transformation stress plateau. Considering the texture of the wire, as seen in Figs. 8 and 9, the wire was not fully homogenous in the unstressed state. The drawing of the wire generates a texture that has aligned the (110) 
Fig. 6 Full Debye-Scherrer diffraction rings at the extreme left, center of bending, and extreme right of the mapped area presented in Fig. 4. Points $A, B$, and $C$ correspond to those shown in Fig. 4 and correlate to $\varepsilon_{11} \approx 90^{\circ}$. As illustrated, the two principal directions, $\varepsilon_{11}$ and $\varepsilon_{22}$, are changing due to the loading mechanism. The initial texture is not changing, but rather the diffraction patterns are rotating since the wire is in a different orientation at different points on the map. This is important to note so that a suitable comparison can be made between the diffraction patterns

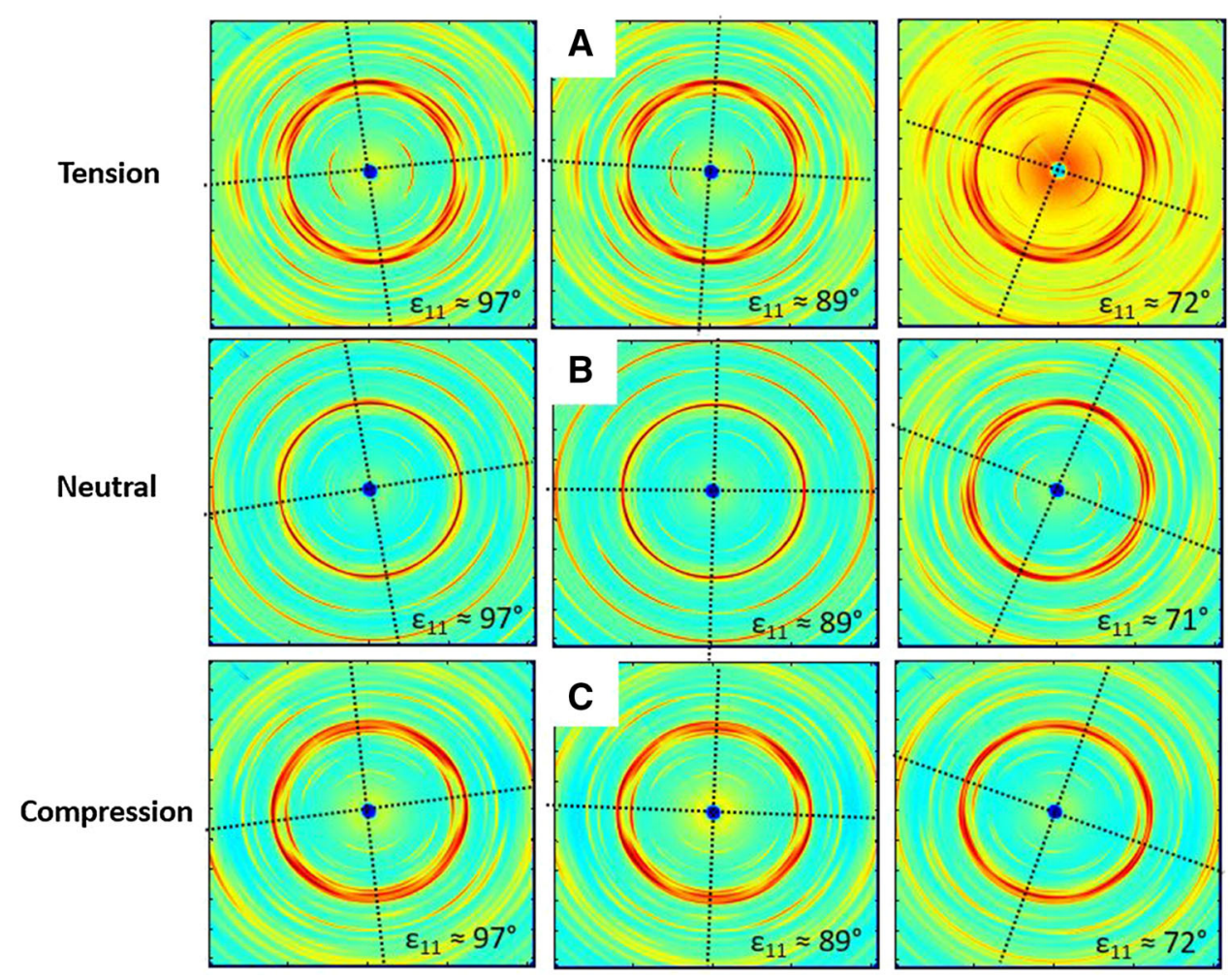

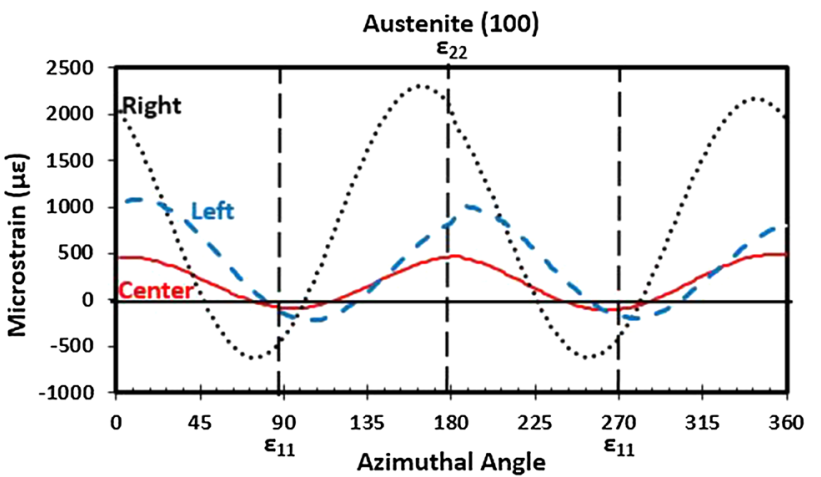

Fig. 7 Measured microstrain vs. azimuthal angle for the austenite (100) planes for the neutral axis at the extreme left (dashed blue curve), center (solid red curve), and extreme right (dotted black curve) positions. Spots correspond to center row of diffraction patterns in Fig. 6. Maximum and minimum strain values were averaged when possible and used as $\varepsilon_{22}$ and $\varepsilon_{11}$, respectively. Notice position of maxima/minima is shifting due to the rotation of the diffraction patterns caused by the wire being oriented differently in position in the diffraction map

austenite planes in the rolling direction $\left(\varepsilon_{11}\right)$ while the (200) austenite peaks align along the transverse direction $\left(\varepsilon_{22}\right)$ similar to what was seen by Hasan et al. [15], although orientation distribution functions were not calculated in our study. Examining the tensile and compressive sides (points $\mathrm{A}$ and $\mathrm{C}$, respectively) shows a striking difference in the texture of the two stress-induced martensitic states. The tensile diffraction patterns look similar to what has been seen by Hasan et al. [15] and Young et al. [16]. All three and the present diffraction pattern at A show the same alignment of the (001) martensite planes along the loading direction or $\varepsilon_{22}$ in this study, the (011) in the approximate $45^{\circ}$ direction and the (100) planes on either side of the transverse or $\varepsilon_{11}$ direction. Showing that both the slightly textured samples and ultra-fined grain samples accommodate the tensile stress in the same manner by using similar martensite variants and twinning structure to create the large macroscopic lengths needed in the wire. A good discussion is given by Daymond et al. [21] in which the authors describe how the NiTi responds to tensile stresses by detwinning and preferentially orienting the grains along the longer $b$ - and $c$-axis, (001) and (010) planes, respectively, of the martensitic crystal to relax the internal stresses in tension and along the much shorter $a$-axis in compression. This is directly observed in the presented diffraction patterns as the (001) peaks align always in the direction of tensile forces, i.e., the $\varepsilon_{22}$ in the tensile side and the $\varepsilon_{11}$ in the compressive side. To further study this directional texturing, the Debye-Scherrer rings were integrated around both the $\varepsilon_{11}$ and $\varepsilon_{22}$ directions for patterns A and $\mathrm{C}$. Though not shown here, the patterns were first integrated over an azimuthal of only $2^{\circ}$ to localize the two principal stress directions. It was found that taking the lineout over plus/minus of $20^{\circ}$ of the principal directions 
Fig. 8 Integrated lineouts showing normalized intensity vs. d-spacing over entire $360^{\circ}$ azimuthal for points $A, B$, and $C$ in Figs. 4 and 6. Points A and $\mathrm{C}$ are fully martensitic, but exhibit different peak intensities associated with different orientations of the martensite phase. Point B is mainly austenite, but with small amounts of R-phase and martensite measureable in the lineouts

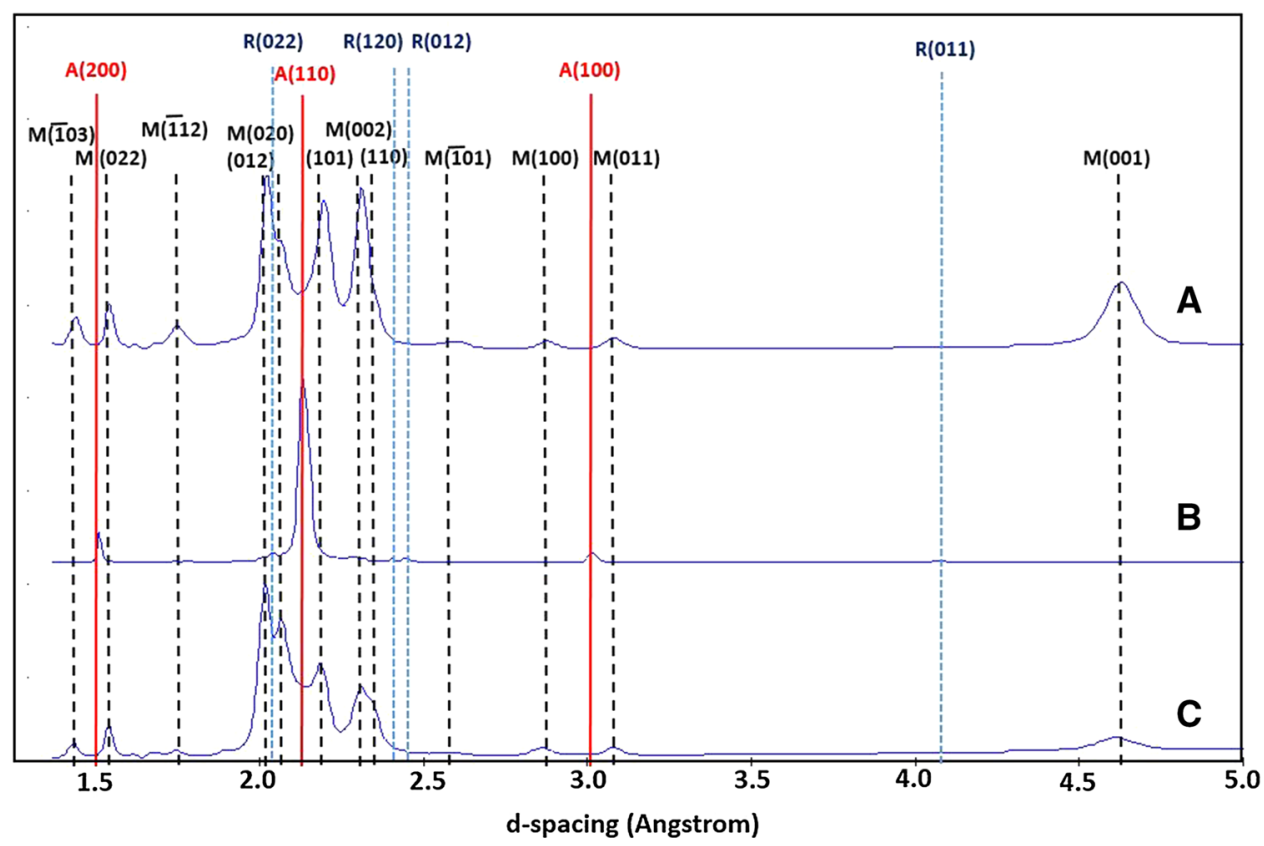

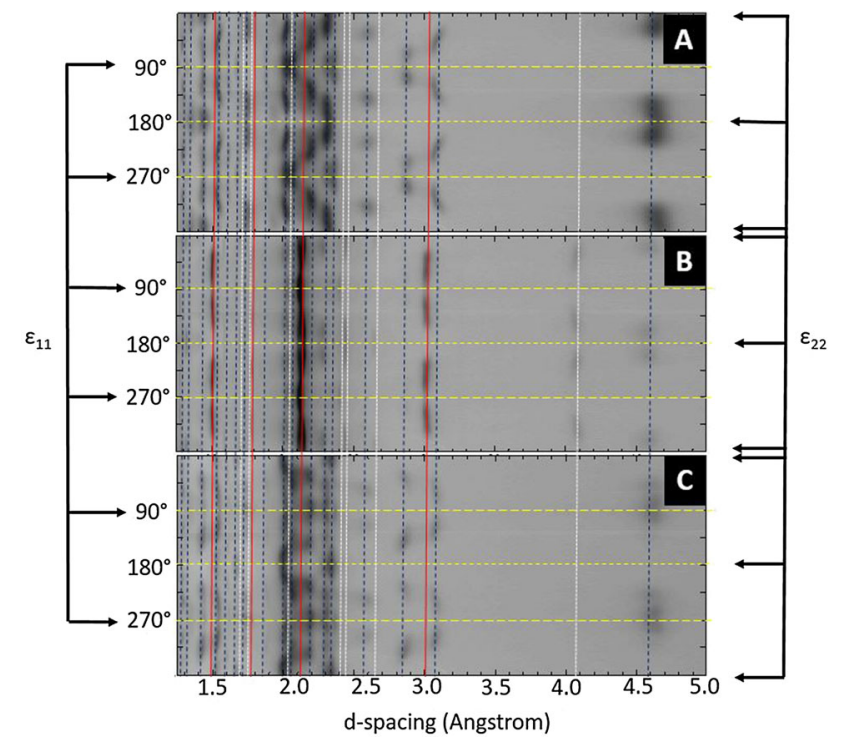

Fig. 9 Cartesian transform of Debye-Scherrer diffraction rings vs. d-spacing for points $A, B$, and $C$ in Figs. 4 and 6 highlight the complex texture differences between the martensite in tension vs. compression. ( $h k l)$ values for phases are not presented to avoid cluttering, but can be observed in Fig. 8. Martensite phase reflections are illustrated with dashed blue lines, austenite with solid red lines, and R-phase with dotted white lines

was almost identical in intensity profiles so these are presented instead to allow for better statistics. Figure 10 shows points $\mathrm{A}$ and $\mathrm{C}$ integrated over the azimuthal range of $70-110^{\circ}, \varepsilon_{11}$, and $160-200^{\circ}, \varepsilon_{22}$. The compressive direction of both patterns is similar with no reflections for the (001) planes present and small reflections from the (100) planes. They differ slightly with the tensile side showing the (020) peak as the max intensity and the compressive side with the max intensity along the (012) planes. However, it looks visually as if both sides of the wire accommodate for the tensile stress state in a similar manner with only slight shifts in relative intensity. The tensile direction is much different, the diffraction pattern from the tensile side is rather simple in comparison than on the compressive side showing only major reflections from the (001) and (002) planes, which are coupled together, meaning the martensite has aligned itself along the $c$-axis

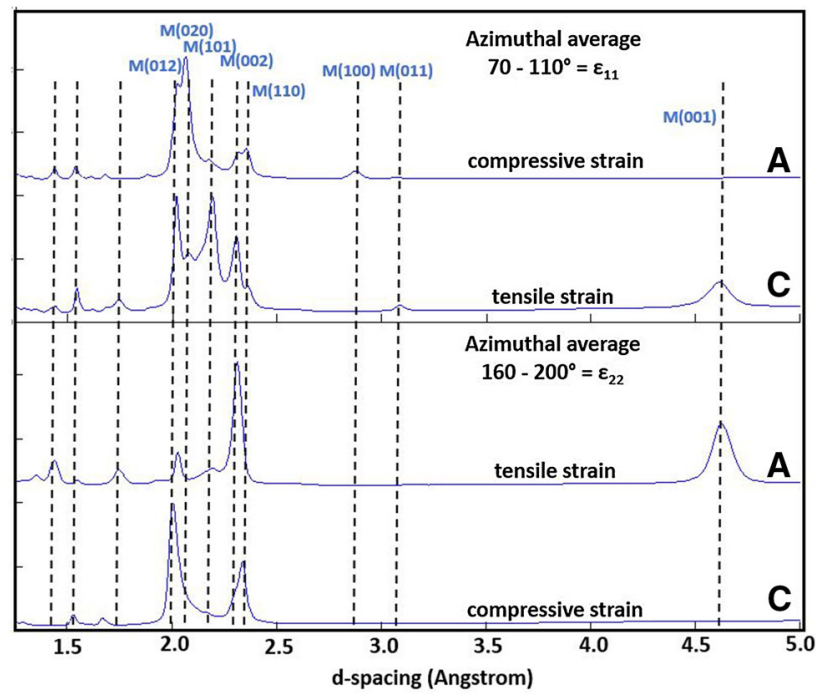

Fig. 10 Integrated lineouts for azimuthal angles $\pm 20^{\circ}$ around the two principal directions, $\varepsilon_{11}$ and $\varepsilon_{22}$, to highlight textural differences between the martensite under tensile and compressive strains in the tensile $(A)$ and compressive $(C)$ side of the NiTi SMA wire 


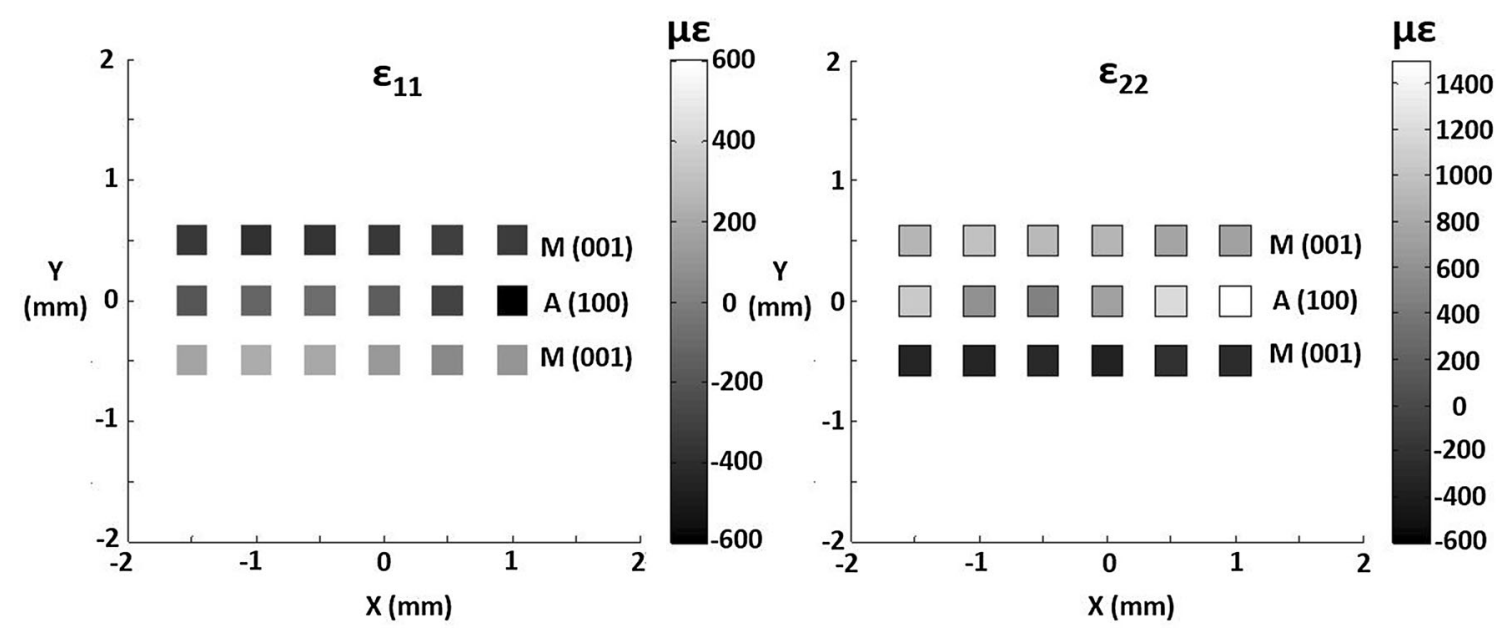

Fig. 11 Calculated microstrain $(\mu \varepsilon)$ values for the major phase of each point in the diffraction map along the two principal directions $\varepsilon_{11}$ and $\varepsilon_{22}$. Strain values are calculated for the martensitic phase from the (001) planes and for the austenitic phase from the (100) planes. The

to help relieve the stress due to tension. On the compressive side, the integrated pattern still shows reflections from the (001) and (002) planes, yet neither is the most intense. The (101) and (012) planes are now the most intense reflections with almost identical intensities. The martensite must find a different way to accommodate this stress state as it is not free to expand in the $\varepsilon_{11}$ direction on the compressive side as it is for the tensile side to expand in the $\varepsilon_{22}$ direction. This can be macroscopically visualized by understanding the stress state in the bent wire. Along the compressive side of the wire, the wire is required to shrink a significant amount in the $\varepsilon_{11}$ direction, it would logically follow, as Daymond et al. [21] describes, that the martensite would choose to orient preferentially along the (100) planes or $a$-axis due to its smaller length. However, by doing so it would align either the $b$-or $c$-axis, which are much longer, in the perpendicular direction. This would cause an elongation in the perpendicular direction, which in normal compression could be fine as the directions perpendicular to loading are not constrained but in the case of pure bending the perpendicular direction is constrained by the interior of the wire. In order to compensate for this, the martensite must find complex orientations and twinning geometries to not only accommodate the compressive state but also the constrained tensile state, which is not an issue along the tensile side of the wire. A more rigorous scientific study is needed to further substantiate these claims and quantify the martensite structure/texture in bending compression and normal uniaxial compression.

Lastly, residual strain values for the martensite (001) and austenite (100) planes are given in Fig. 11 depending on the major phase present. The column of values extending from $x=-0.5 \mathrm{~mm}$ correspond to the spots A, high strain associated with the austenite $(100)$ at position $(1,0)$ is because this point is in the transformation zone. This can be seen in Fig. 6 as the full Debye-Scherrer diffraction pattern contains large amounts of both austenite and martensite

$\mathrm{B}$, and $\mathrm{C}$ in the previous Figs. 4, 6, 8, 9, and 10. As expected, the tensile side of the wire along the $\varepsilon_{22}$ direction is under a tensile strain while the compression side is under compressive strain. The Poisson effect is observed along the $\varepsilon_{11}$ direction for both the tensile and compressive sides of the wire, as they are under compressive and tensile strains, respectively. The martensite (001) planes are under the greatest strains at $-1 \mathrm{~mm}$ along the $x$-axis and drop-off when moving to either the left or right with a maximum variance of approximately $250 \mu \varepsilon$, associated with the $\varepsilon_{22}$ on the tensile side. It should be noted that due to the texture of the (001) martensite peaks, only the $\varepsilon_{22}$ and $\varepsilon_{11}$ directions for the tensile and compressive sides, respectively, are directly measureable. This means that the other principal direction is merely an estimate made by extrapolation and should be taken with caution. Still the trend in the strain values is apparent with the values dropping off on both sides of the $x=-1 \mathrm{~mm}$ position. However, it seems that the wire under $12 \%$ surface strain is accommodating the bending stress rather successfully through the martensitic transformation and detwinning mechanisms as evident by the almost constant strain values, but could be approaching its limit as the lattice strains are beginning to slightly rise along the wire position. The strains along the neutral axis, mainly austenitic, however, are changing quite dramatically as a function of position. The austenite (100) peak is under the lowest strains at the $x=-0.5 \mathrm{~mm}$, point $\mathrm{B}$, which is expected considering this is closest to the center of bending radius and along the neutral axis of the wire under bending. The residual strain is still approximately $475 \mu \varepsilon$ at the position and it is interesting to point out that this is the approximate $\mu \varepsilon$ value, $380 \mu \varepsilon$ for the austenite (100) planes where Young et al. [16] start to see a 
measureable appearance of both the R-phase and martensite. As the position is shifted either left or right, the lattice strains increase in a parabolic nature. The strain value on the far right, $x=1 \mathrm{~mm}$, is much higher than the other values, approximately $2000 \mu \varepsilon$, and can be attributed to the transformation zone. This can also be seen visually in Fig. 5 with close examination of the full Debye-Scherrer ring, and noticing that it shows high intensities of both the austenite and martensite phase reflections. The measured diffraction spot has now shifted just far enough off the neutral axis to begin the stress-induced transformation. Upon closer examination, it can be shown that the transformation is evolving in the same manner as the tensile side of the wire, which again is expected.

\section{Discussion of Results}

The obvious cracking exhibited by the $30 \%$ surfacestrained sample along the compressive side of the wire is evidence of the eventual failure of pseudoelastic NiTi wires in bending applications. At the present time, it is not established at what precise strain values, either lattice or macroscopic, when the wire fails and begins to crack but it is localized on the compressive side due to the strong anisotropic tension-compression deformation behavior exhibited by NiTi alloys [24]. However, when considering fatigue life, these micro-cracks will propagate through the NiTi SMA wire under tensile and compressive loading though the mechanisms are most likely different. It was shown by Gollerthan et al. [25] using compact tension specimens that pseudoelastic NiTi SMA compact tension samples will transform around the crack tip to the martensitic phase then the crack will propagate through the structure leaving a retransformed region of austenite in its wake due to the now unstressed state. In a later publication Young et al. [22] mapped the phase fraction and lattice strains around a crack and showed that the highest volume fraction of martensite occurs right at the crack tip and large tensile strains in the loading direction $2 \mathrm{~mm}$ above and below the crack exist. This type of behavior would occur upon unloading and reloading of the wire in cycled bending applications. The cracks would initiate and grow in the compressive state due to the volume expansion associated with the stress-induced transformation in compression. Then as the wire is unbent/unloaded, the material would transform back to the austenitic state now with small micro-cracks. As the material is reloaded in the opposite direction, the regions near these micro-crack would transform first due to the stress concentration and then propagate through the structure, but now the opposite side of the wire could also potentially develop new cracks. This loading sequence would continue with the cracks now growing from the surface to the center of the wire until the eventual failure of the wire.

More work is needed to full understand the exact mechanisms for crack initiation and crack propagation on the compressive side of bending in pseudoelastic NiTi SMA wires. Bending fatigue experiments in combination with SR-XRD mapping measurements is a good approach to understand the deformation behavior and fatigue life of these wires. In future experiments, it is recommended that the mapping area be finer and more comprehensive of the complex texture and stress state exhibited in the wire with special attention on the compressive side before, at the point of, and after crack initiation has begun. Examining the phase fraction and lattice strains at the onset of crack initiation will help to elucidate the fundamental problems and doing so once a crack is initiated in the immediate areas on both the tensile and compressive side will lead to a better understanding of propagation mechanisms.

\section{Conclusion}

In the present study, microstructure, bending surface texture, and residual strains were examined using SEM and SR-XRD measurements of austenitic pseudoelastic NiTi SMA wires were investigated during simple bending. From this study, we were able to determine that the mechanism for failure of these wires is due to micro-crack initiation localized on the compressive side of the wire. Further examination of a $12 \%$ surface strain wire was performed using SR-XRD measurements before the initiation of these micro-cracks. This revealed that the tensile and compressive sides of the NiTi SMA wire deform anisotropically as evident by the strong texture difference in the surface and SR-XRD patterns. The micro-cracks are likely formed due to the volume expansion associated with the martensitic transformation along the axis perpendicular to bending radius, described as $\varepsilon_{11}$ in the present study. Once these cracks have initiated, they act as nucleation points for the martensitic transformation to occur in either tension or compression and will propagate towards the center of the wire regardless of the direction of bending, i.e., neither the tensile or compressive side of the bending axis.

Acknowledgments The authors would like to thank Scott Robertson (NDC, now at Medtronic) for supplying the SMA materials, Ying Qiu for help with electropolishing, and beamline scientists Jon Almer and Jun-Sang Park for help with the experiments at the Advanced Photon Source (APS) and for providing the preliminary MATLAB code to analyze the diffraction patterns. This research used resources of the Advanced Photon Source, a U.S. Department of Energy (DOE) Office of Science User Facility operated for the DOE Office of Science by Argonne National Laboratory under Contract No. DE-AC0206CH11357. This project was financially supported by UNT's ROP grant. 


\section{References}

1. Otsuka K, Ren X (2005) Physical metallurgy of Ti-Ni-based shape memory alloys. Prog Mater Sci 50:511-678

2. Buehler WJ, Gilfrich J, Wiley R (1963) Effect of low-temperature phase changes on the mechanical properties of alloys near composition TiNi. J Appl Phys 34:1475-1477

3. Sun L, Huang WM, Ding Z, Zhao Y, Wang CC, Purnawali H, Tang C (2012) Stimulus-responsive shape memory materials: a review. Mater Des 33:577-640

4. Melton K, Mercier O (1981) The mechanical properties of NiTibased shape memory alloys. Acta Metall 29:393-398

5. Es-Souni M, Es-Souni M, Fischer-Brandies H (2002) On the properties of two binary NiTi shape memory alloys. Effects of surface finish on the corrosion behaviour and in vitro biocompatibility. Biomaterials 23:2887-2894

6. Tan L, Dodd R, Crone W (2003) Corrosion and wear-corrosion behavior of NiTi modified by plasma source ion implantation. Biomaterials 24:3931-3939

7. Imbeni V, Martini C, Prandstraller D, Poli G, Trepanier C, Duerig T (2003) Preliminary study of micro-scale abrasive wear of a NiTi shape memory alloy. Wear 254:1299-1306

8. Trepanier C, Tabrizian M, Yahia LH, Bilodeau L, Piron DL (1998) Effect of modification of oxide layer on NiTi stent corrosion resistance. J Biomed Mater Res 43:433-440

9. Rondelli G (1996) Corrosion resistance tests on NiTi shape memory alloy. Biomaterials 17:2003-2008

10. Van Humbeeck J (1999) Non-medical applications of shape memory alloys. Mater Sci Eng A 273:273-275

11. Norwich D, Fasching A (2009) A study of the effect of diameter on the fatigue properties of NiTi wire. J Mater Eng Perform 18:558-562

12. Eggeler G, Hornbogen E, Yawny A, Heckmann A, Wagner M (2001) Structural and functional fatigue of NiTi shape memory alloys. Mater Sci Eng A 378:24-33

13. James B, Foulds J, Eiselstein L (2005) Failure analysis of NiTi wires used in medical applications. J Fail Anal Prev 5:82-87

14. Schmahl WW, Khalil-Allafi J, Hasse B, Wagner M, Heckmann A, Somsen C (2004) Investigation of the phase evolution in a super-elastic NiTi shape memory alloy (50.7 at.\%Ni) under extensional load with synchrotron radiation. Mater Sci Eng A 378:81-85

15. Hasan M, Schmahl WW, Hackl K, Heinen R, Frenzel J, Gollerthan S, Eggeler G, Wagner M, Khalil-Allafi J, Baruj A (2008) Hard X-ray studies of stress-induced phase transformations of superelastic NiTi shape memory alloys under uniaxial load. Mater Sci Eng A 481-482:414-419

16. Young M, Wagner M, Frenzel J, Schmahl W, Eggeler G (2010) Phase volume fractions and strain measurements in an ultrafinegrained NiTi shape-memory alloy during tensile loading. Acta Mater 58:2344-2354

17. Cai S, Schaffer JE, Daymond MR, Yu C, Ren Y (2014) Effect of heat treatment temperature on nitinol wire. Appl Phys Lett 105(7):071904

18. Wagner M, Sawaguchi T, Kausträter G, Höffken D, Eggeler G (2004) Structural fatigue of pseudoelastic NiTi shape memory wires. Mater Sci Eng A 378:105-109

19. Young M, Almer J, Daymond M, Haeffner D, Dunand D (2007) Load partitioning between ferrite and cementite during elastoplastic deformation of an ultrahigh-carbon steel. Acta Mater 55:1999-2011

20. Hammersley A (1997) FIT2D: an introduction and overview, European Synchrotron Radiation Facility Internal Report ESRF97HA02T

21. Daymond M, Young M, Almer J, Dunand D (2007) Strain and texture evolution during mechanical loading of a crack tip in martensitic shape-memory NiTi. Acta Mater 55:3929-3942

22. Young M, Gollerthan S, Baruj A, Frenzel J, Schmahl WW, Eggeler G (2013) Strain mapping of crack extension in pseudoelastic NiTi shape memory alloys during static loading. Acta Mater 61:5800-5806

23. Xue D, Zhou Y, Ren X (2011) The effect of aging on the B2-R transformation behaviors in Ti-51at\% $\mathrm{Ni}$ alloy. Intermetallics 19:1752-1758

24. Orgeas L, Favier D (1995) Non-symmetric tension-compression behaviour of NiTi alloy. J Phys IV 5:C8, 605-610

25. Gollerthan S, Young ML, Neuking K, Ramamurty U, Eggeler G (2009) Direct physical evidence for the back-transformation of stress-induced martensite in the vicinity of cracks in pseudoelastic NiTi shape memory alloys. Acta Mater 57:5892-5897 